\title{
THE UNIVERSAL COMPACT SUBUNITHETIC SEMIGROUP
}

JOHN A. HILDEBRANT

Compact subunithetic semigroups have been studied in [3] and [4], and related results can be found in [5], [6], and [7].

The structure of compact subunithetic semigroups is completely determined in this paper by exhibiting a universal compact subunithetic semigroup in the continuous homomorphism sense and a universal compact unithetic semigroup in the embedding sense. Generalizations of some of the results of [4] to include nonabelian semigroups are obtained.

If $S$ is a compact [uniquely] divisible semigroup and $x \in S$, then there exists a [unique] minimal compact divisible subsemigroup $S(x)$ of $S$ which contains $x$. Moreover, each such $S(x)$ is subunithetic [unithetic]. Thus the study of the structure of compact subunithetic semigroups is essential to the study of compact divisible semigroups.

Notation. The following notation will be used throughout this paper:

1. $N=$ set of all positive integers;

2. $Q=$ discrete additive semigroup of positive rationals;

3. $I=[0,1]$ with usual multiplication and topology;

4. $\Sigma=a$-adic solenoid with $a=(2,3, \cdots)[2$, p. 114];

5 . $\Sigma^{*}=$ universal compact solenoidal group $[2,25.19]$;

6. $\Phi=$ universal compact solenoidal semigroup [6, II]

A semigroup $S$ is said to be [uniquely] divisible if for each $y \in S$ and each $n \in N$, there exists an [unique] element $x \in S$ such that $y=x^{n}$. A topological semigroup $T$ is said to be subunithetic if $T$ contains a dense homomorphic image of $Q$ (Note that $T$ is divisible and abelian). A subunithetic semigroup $T$ is said to be unithetic if $T$ is uniquely divisible. If $T$ is a unithetic semigroup and $\sigma: Q \rightarrow T$ is a homomorphism such that $\sigma(Q)$ is dense in $T$, then the element $x=\sigma(1)$ is called a unithetic generator of $T$. (Note that the rational powers of $x$ are dense in $T$.)

If $S$ is a uniquely divisible topological semigroup and $x \in S$, then the subsemigroup $[x]=\left\{x^{r}: r \in Q\right\}-$ (closure in $S$ ) is the unithetic subsemigroup of $S$ generated by $x$. Note that $S$ is unithetic if and only if $S=[x]$ for some $x \in S$.

Received by the editors October 24, 1968. 
The Bohr (or almost periodic) compactification of a topological semigroup $S$ is a pair $(B, \beta)$ such that:

(i) $B$ is a compact semigroup;

(ii) $\beta: S \rightarrow B$ is a continuous homomorphism of $S$ into $B$ such that $\beta(S)$ is dense in $B$; and

(iii) if $f: S \rightarrow T$ is a continuous homomorphism of $S$ into a compact semigroup $T$, then there exists a unique continuous homomorphism $f^{*}: B \rightarrow T$ such that $f^{*} \beta=f$.

The existence and uniqueness of the Bohr compactification can be obtained as a consequence of the adjoint functor theorem [8]. Related results can be found in [1]. The semigroup $\Phi$ is the Bohr compactification of the additive semigroup $R$ of nonnegative reals with the usual topology, and $\Sigma^{*}$ is the Bohr compactification of the group $R \cup(-R)$. Both $\Phi$ and $\Sigma^{*}$ are unithetic. One of the most essential results in this paper is that $\Sigma^{*} \times \Phi$ is the Bohr compactification of $Q$.

LemMA 1. Let $h \in \Sigma^{*}$ and $\left\{p_{n}\right\}$ a sequence of positive prime integers such that $(n+1) !<p_{n}$ and $p_{n}+n<p_{n+1}$ for each $n \in N$. Then there exists $g \in \Sigma^{*}$ such that $\left\{g^{1 / p_{n}}\right\}$ converges to $h$.

Proof. We first prove this result for $\Sigma$. Note that $\Sigma$ is the projective limit of copies of the circle group with bonding sequence $(2,3,4, \cdots)$. Let $h_{0}=\left(h_{1}, h_{2}, \cdots\right)$ be an element of $\Sigma$. We will construct $g_{0}=\left(g_{1}, g_{2}, \cdots\right)$ in $\Sigma$ inductively such that $\left\{g_{0}^{1 / p_{n}}\right\}$ converges to $h_{0}$. Let $g_{1}=\cdots=g_{p_{1}-1}=\exp (2 \pi i)$. Let $n \in N$, and suppose $g_{p_{n}-1}=\exp \left(2 \pi i S_{n}\right)$ has been defined for some real number $S_{n}$. Now $h_{n}=\exp \left(2 \pi i t_{n}\right)$ for some real number $t_{n}$. Then, since $h_{0} \in \Sigma$, there exist real numbers $t_{1}, t_{2}, \cdots, t_{n-1}$ such that $(j+1) ! t_{j+1}=t_{1}$ and $h_{j}=\exp \left(2 \pi i t_{j}\right)$ for $j=1,2, \cdots, h-1$. Let

$$
r_{n}=\frac{t_{1} p_{n}-S_{n}\left(p_{n}-1\right) !}{n ! p_{n}} .
$$

Then there exists an integer $k_{n}$ such that $p_{n} r_{n}-1 \leqq-k_{n} \leqq p_{n} r_{n}+1$. Since $p_{n}$ is prime, $\left(\left(p_{n}-1\right) !+1\right) / p_{n}$ is an integer, and hence $m_{n}=k_{n}\left(\left(p_{n}-1\right) !+1\right) / p_{n}$ is an integer. Thus

$$
t_{1}-n ! / p_{n} \leqq\left(S_{n}+h ! k_{n}\right) \cdot\left(p_{n}-1\right) ! / p_{n}-h ! m_{n} \leqq t_{1}+n ! / p_{n} .
$$

It now follows that

$$
\begin{aligned}
t_{j+1}-\frac{n !}{p_{n}(j+1) !} & \leqq \frac{\left(S_{n}+n ! k_{n}\right)\left(p_{n}-1\right) !}{p_{n}(j+1) !}-\frac{n ! m_{n}}{(j+1) !} \\
& \leqq t_{j+1}+\frac{n !}{p_{n}(j+1) !}
\end{aligned}
$$


and $n ! m_{n} /(j+1) !$ is an integer for $j=0,1,2, \cdots, n-1$. Define

$$
g_{p_{n}+j}=\exp \left(\frac{2 \pi i\left(S_{n}+n ! k_{n}\right)\left(p_{n}-1\right) !}{\left(p_{n}+j\right) !}\right) \quad \text { for } j=0,1, \cdots, n-1 \text {, }
$$

and define $g_{j}$ such that $g_{j}^{j}=g_{j-1}$ for $j=p_{n}+n, \cdots, p_{n+1}-1$. Thus $g_{0}$ is defined inductively such that $g_{0} \in \Sigma$. Now

and

$$
\begin{gathered}
g_{0}^{1 / p_{n}}=\left(g_{p_{n}}^{p_{n} ! / p_{n}}, \cdots, g_{p_{n}+j}^{\left(p_{n}+j\right) ! / p_{n}(j+1) !}, \cdots\right) \\
g_{p_{n}}^{\left(p_{n}+j\right) ! / p_{n}(j+1) !}=\exp \left(\frac{2 \pi i\left(S_{n}+n ! k_{n}\right)\left(p_{n}-1\right) !}{p_{n}(j+1) !}\right) \\
\text { for } j=0,1, \cdots, n-1 .
\end{gathered}
$$

Since $\left\{n ! / p_{n}\right\}$ converges to 0 , it follows from the last inequality above that $\left\{g_{0}^{1 / p_{n}}\right\}$ converges to $h_{0}$. This proves the result for $\Sigma$.

Let $\Sigma_{\alpha}=\Sigma$ for $\alpha \in I$. Then $\Sigma^{*}=\prod\left\{\Sigma_{\alpha}: \alpha \in I\right\}$. Let $\pi_{\alpha}: \Sigma^{*} \rightarrow \Sigma_{\alpha}$ be the projection map, and $h_{\alpha}=\pi_{\alpha}(h)$ for each $\alpha \in I$. Then there exists $g_{\alpha} \in \Sigma_{\alpha}$ such that $\left\{g_{\alpha}^{1 / p_{n}}\right\}$ converges to $h_{\alpha}$ for each $\alpha \in I$. Let $g \in \Sigma^{*}$ such that $\pi_{\alpha}(g)=g_{\alpha}$ for each $\alpha \in I$. Then $\left\{g^{1 / p_{n}}\right\}$ converges to $h$, and the proof of the lemma is complete.

\section{THEOREM 2. The Bohr compactification of $Q$ is $\Sigma^{*} \times \Phi$.}

Proof. Let $x$ be a unithetic generator of $\Phi$ and 1 the identity of $\Phi$. Let $h$ be a unithetic generator of $\Sigma^{*}$ and $\left\{p_{n}\right\}$ a sequence of positive prime integers such that $(n+1) !<p_{n}$ and $p_{n}+h<p_{n+1}$ for each $n \in N$. Then, by Lemma 1 , there exists $g \in \Sigma^{*}$ such that $\left\{g^{1 / p_{n}}\right\}$ converges to $h$. Thus $\left\{(g, x)^{1 / p_{n}}\right\}$ converges to $(h, 1)$. Since $h$ is a unithetic generator of $\Sigma^{*}$, it follows that $\Sigma^{*} \times\{1\} \subset[(g, x)]$. Let $(a, b) \in \Sigma^{*} \times \Phi$. Then there exists $u \in \Sigma^{*}$ such that $(u, b) \in[(g, x)]$. Let $u^{-1}$ denote the inverse of $u$ in $\Sigma^{*}$. Then $\left(u^{-1} a, 1\right) \in[(g, x)]$ and hence $(a, b)=(u, b)$ $\cdot\left(u^{-1} a, 1\right)$ is in $[(g, x)]$. It follows that $\Sigma^{*} \times \Phi$ is unithetic, and that $(g, x)$ is a unithetic generator of $\Sigma^{*} \times \Phi$. Define $\beta: Q \rightarrow \Sigma^{*} \times \Phi$ by $\beta(r)$ $=(g, x)^{r}$ for each $r \in Q$. Then $\beta$ is a homomorphism such that $\beta(Q)$ is dense in the compact semigroup $\Sigma^{*} \times \Phi$. Let $S$ be a compact subunithetic semigroup, $e$ the identity of $S$, and $f: Q \rightarrow S$ a homomorphism such that $f(Q)$ is dense in $S$. Then $S$ contains a compact solenoidal subsemigroup $T$ such that $S=H(e) T$ [4, Theorem 2.2]. Define $\phi: H(e) \times T \rightarrow S$ by $\phi(s, t)=s t ; s \in H(e), t \in T$. Then $\phi$ is a continuous onto homomorphism since $S$ is abelian. Let $s_{0} \in H(e)$ and $t_{0} \in T$ such that $\phi\left(s_{0}, t_{0}\right)=f(1)$. Then there exist continuous onto homomorphisms $\alpha: \Sigma^{*} \rightarrow H(e)$ and $\lambda: \Phi \rightarrow T$ such that $\alpha(g)=s_{0}$ and $\lambda(x)=t_{0}[4]$. Define $f_{1}^{*}: \beta(Q) \rightarrow S$ by $f_{1}^{*} \beta(r)=\phi\left(\alpha\left(g^{r}\right), \lambda\left(x^{r}\right)\right) ; r \in Q$. Then, since $\beta(Q)$ 
is dense in $\Sigma^{*} \times \Phi, f_{1}^{*}$ has a unique extension to $f^{*}: \Sigma \times \Phi \rightarrow S$, and $f^{*} \beta=f$. It follows that $\left(\Sigma^{*} \times \Phi, \beta\right)$ is the Bohr compactification of $Q$.

Corollary 3. A compact semigroup is subunithetic if and only if it is a continuous homomorphic image of $\Sigma^{*} \times \Phi$.

Corollary 4. Let $S$ and $T$ be compact semigroups. Then $S \times T$ is [sub-] unithetic if and only if $S$ and $T$ are [sub-] unithetic and either $S$ or $T$ is a group.

Corollary 5. The semigroup $\left(\left(\Sigma^{*} \times I\right) /\left(\Sigma^{*} \times\{0\}\right)\right) \times \Sigma^{*}$ is unithetic.

THEOREM 6. Let $S$ be a compact semigroup. Then these are equivalent:

(i) $S$ is [uniquely] divisible:

(ii) each component of $S$ is a [uniquely] divisible subsemigroup of $S$; and

(iii) if $x \in S$, then there exists a [unique] subsemigroup $S(x)$ of $S$, which is minimal with respect to being a compact divisible subsemigroup of $S$ containing $x$; and each such $S(x)$ is [unithetic] subunithetic.

Proof. The proof follows from the fact that $\Sigma^{*} \times \Phi$ is connected, and, if $S$ is divisible, and $x \in S$, then there exists a continuous homomorphism $\lambda: \Sigma^{*} \times \Phi \rightarrow S$ such that $\lambda(y)=x$, for some unithetic generator $y$ of $\Sigma^{*} \times \Phi$.

Notation. If $S$ is a semigroup, then $E(S)$ denotes the set of idempotent elements of $S$.

Corollary 7. Let $S$ be a compact totally disconnected semigroup. Then $S$ is divisible if and only if $S=E(S)$.

Corollary 8. A finite semigroup $S$ is divisible if and only if $S=E(S)$.

COROllary 9. Let $S$ be a compact semigroup. Then each element of $S \backslash E(S)$ lies on a [unique usual] thread in $S$ if and only if $S$ is [uniquely] divisible and has degenerate subgroups.

Theorem 10. Let $S$ be a compact unithetic semigroup. Then $S$ is topologically isomorphic to a subsemigroup of $\left(\left(\Sigma^{*} \times I\right) /\left(\Sigma^{*} \times\{0\}\right)\right) \times \Sigma^{*}$.

Proof. Let 1 denote the identity of $S, K$ the minimal ideal of $S$, and $h: H(1) \rightarrow \Sigma^{*}$ and $k: K \rightarrow \Sigma^{*}$ injections. (See [3, Theorem 2.3 and Theorem 3.1].) Let $\gamma: S / K \rightarrow(H(1) \times I) /(H(1) \times\{0\})$ be a topological isomorphism. (See [3, Theorem 3.4].) Let $j: I \rightarrow I$ be the identity map, $\alpha: H(1) \times I \rightarrow(H(1) \times I) /(H(1) \times\{0\})$ the natural maps, and $\lambda: \Sigma^{*} \times I \rightarrow\left(\Sigma^{*} \times I\right) /\left(\Sigma^{*} \times\{0\}\right)$ the natural map. Then there exists an induced injection $\phi$ such that the diagram: 


$$
\begin{gathered}
(H(1) \times I) /(H(1) \times\{0\}) \stackrel{\phi}{\rightarrow}\left(\Sigma^{*} \times I\right) /\left(\Sigma^{*} \times\{0\}\right) \\
\alpha \uparrow \\
H(1) \times I \longrightarrow \lambda \\
\rightarrow \Sigma^{*} \times I
\end{gathered}
$$

commutes. Let $e$ denote the identity of $K$ and define $\sigma: S \rightarrow(S / K) \times K$ by $\sigma(x)=(\psi(x), e x) ; x \in S$, where $\psi: S \rightarrow S / K$ is the natural map. Then $\sigma$ is an injection. Let $\rho: \Sigma^{*} \rightarrow \Sigma^{*}$ be the identity map. Then

$$
S \stackrel{\sigma}{\rightarrow}(S / K) \times K \stackrel{\gamma \times k}{\rightarrow} \frac{(H(1) \times I)}{(H(1) \times\{0\})} \times \Sigma^{*} \stackrel{\phi \times \rho}{\rightarrow} \frac{\left(\Sigma^{*} \times I\right)}{\left(\Sigma^{*} \times\{0\}\right)} \times \Sigma^{*}
$$

defines the desired injection, and the proof of the theorem is complete.

\section{REFERENCES}

1. J. F. Berglund and K. H. Hofmann, Compact semitopological semigroups and weakly almost periodic functions, Lecture Notes, Springer-Verlag, New York, 1967.

2. E. Hewitt and K. A. Ross, Abstract harmonic analysis, Academic Press, New York, 1963.

3. J. A. Hildebrant, On compact unithetic semigroups, Pacific J. Math., 2 (1967), 265-273.

4. - On compact divisible abelian semigroups, Proc. Amer. Math. Soc., 19 (1968), 405-410.

5. K. H. Hof mann, Topologische Halbgruppen mit dichter submonoger Unterhalbgruppe, Math Z., 74 (1960), 232-276.

6. K. H. Hofmann and P. S. Mostert, Elements of compact semigroups, Merill, Columbus, Ohio, 1966.

7. Anne Lester (Hudson), Some semigroups on the two-cell, Proc. Amer. Math. Soc. 10 (1959), 648-655.

8. B. Mitchell, Theory of categories, Academic Press, New York, 1965.

Louisiana State University, Baton Rouge 